\title{
Hirayama Disease: Magnetic Resonance Imaging Manifestations and Single Centre Experience
}

Rathore P*, Raj G, Yadav R and Singh A

Department of Radiodiagnosis, Dr Ram Manohar Lohiya

Institute of Medical Sciences, Lucknow, India

*Corresponding author: Rathore P, Department of Radiodiagnosis, Dr. Ram Manohar Lohiya Institute of Medical Sciences, Lucknow, India-226010

Received: March 15, 2021; Accepted: April 16, 2021; Published: April 23, 2021

\author{
Abstract \\ Purpose: A retrospective study to evaluate various MR imaging spectrums \\ of clinically diagnosed cases of Hirayama disease.
}

Materials and Method: This study included a review of images of 13 patients on the hospital information system who underwent imaging evaluation for suspected Hirayama disease on 3 Tesla MRI scanner in postcontrast neutral and flexion positions. Images were evaluated for the following findings: Localised lower cervical cord atrophy, asymmetric cord flattening, Abnormal cervical curvature, an Increased signal on T2WS, Loss of attachment between posterior dura and lamina, presence of flow void with enhancement.

Observations: Lower cervical cord atrophy, anterior dural shift with flexion, and postcontrast enhancement of epidural component was found in thirteen out of thirteen patients (100\%), followed by the hyperintense signal in T2WS in the cord which was found in 11 patients. Loss of attachment of posterior dura and subjacent lamina and abnormal curvature of the spine was found in 9 out of thirteen patients (91.3\%), prominent epidural flow void was found in eight out of thirteen patients (61.5\%), the thoracic extension of enhancing epidural component was found in five patients (38.4\%).

Keywords: Magnetic resonance imaging; Neutral and flexion; Hirayama disease; Amyotrophic lateral sclerosis; Juvenile spinal muscular atrophy

\section{Introduction}

Hirayama disease also known as nonprogressive juvenile spinal muscular atrophy or sobue disease is a rare disease characterized by unilateral/ b/l asymmetrical muscle weakness of C8-T1 distribution [1]. It was first reported in 1959 [2] as unilateral muscular atrophy. It is most commonly seen in Asian countries like India and Japan [3] as well as in Europe and North America. The Peak age of onset is 1517 Yrs and male preponderance is seen [4]. The main clinical features are wasting of muscles of the hand and forearm with sparing of brachioradialis. Onset is insidious with clinical arrest for several years [5]. It is unilateral in most cases, asymmetrically bilateral in some, and rarely symmetrical [6]. Microcirculatory damage in anterior spinal artery territory caused by repeated flexion is the hallmark in pathological studies [1]. A spectrum of diagnostic features is described for this entity. In this study, we tried to establish various MRI findings in this rare disease entity.

\section{Materials and Methods}

This is a retrospective study on thirteen patients diagnosed with Hirayama disease to evaluate MR imaging spectrum. The pre and post-contrast images in neutral and flexion positions were evaluated and correlated with clinical findings. All were males between the age of 15-30 Yrs. Clinical findings in most of the patients were insidious onset weakness and atrophy in one upper limb or asymmetrically in both the limb limited to C7, C8 and, T1 myotomes. The disease had a benign course with arrest in progression after a few years.

\section{Technical consideration}

MRI was done in all patients on 16 channels 3T MR (GE Healthcare) in neutral and flexion position with the postcontrast study. MRI protocol included sagittal T1WS, sagittal, and axial T2WS in a neutral position, and sagittal T2WS and postcontrast images in the flexion position. Postcontrast transverse and sagittal T1WS in flexion and extension were obtained in all cases. Section thickness was $3.5 \mathrm{~mm}$ with a $1 \mathrm{~mm}$ gap for all images.

The lower cervical cord was defined as the cord between C4 and C7. The following features were evaluated: Localised lower cervical cord atrophy, asymmetric cord flattening, abnormal cervical curvature, loss of attachment between the posterior dural sac and lamina, anterior shifting of posterior wall of cervical dura, and enhancing epidural component with flow voids. Localized cord atrophy was defined as the thinning of the cord as compared to that below the affected level [7].

Asymmetric cord flattening was defined on transverse MR images, an elliptical spinal cord was considered as normal while the pear-shaped and triangular cord was considered as asymmetrical cord flattening. Cervical curvature was measured according to classification is given by Guigui et al. [8] and batzdorf [9] which states, Cervical curvature was measured according to the relationship of the dorsal aspect of vertebral bodies $\mathrm{C} 3$ through $\mathrm{C} 6$ crosses the line from C2 through C7. Abnormal curvature is a curvature in which part or all of the dorsal aspect of vertebral bodies C3 through C6 meet or cross the line from C2 through C7 [7]. 


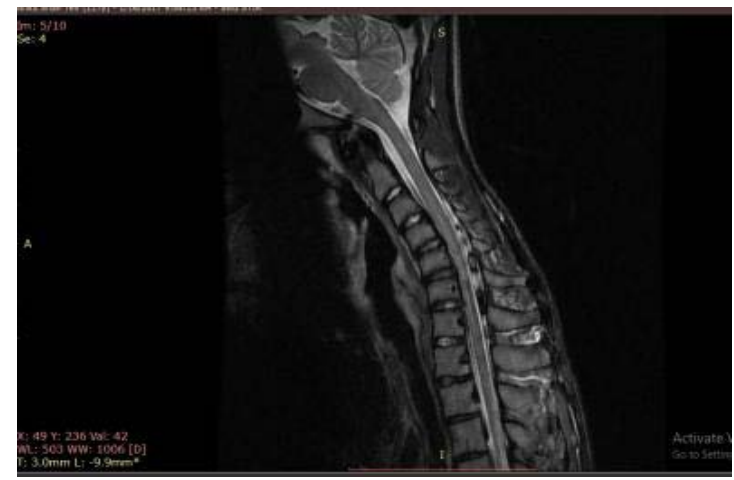

Figure 1: Flexion STIR image showing anterior displacement of posterio dura with epidural flow voids. Block arrow: flow void, arrow: posterior dura.

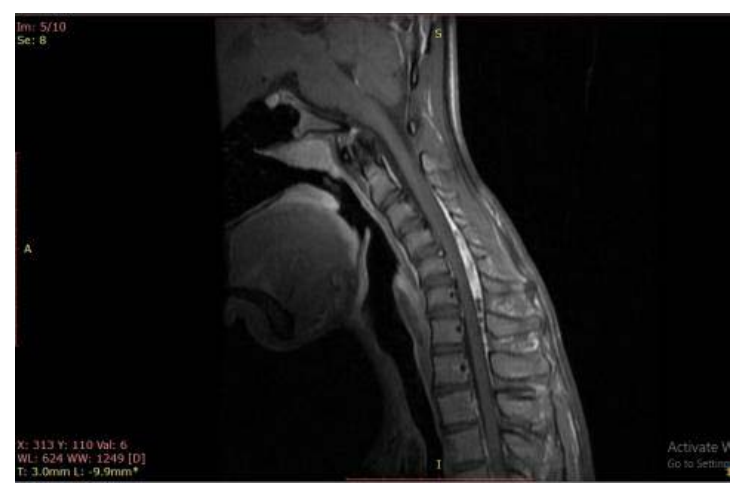

Figure 2: Sagittal T1FS postcontrast image shows enhancement of posterior epidural space with anterior displacement of posterior dura. Arrow: enhancing epidural space.

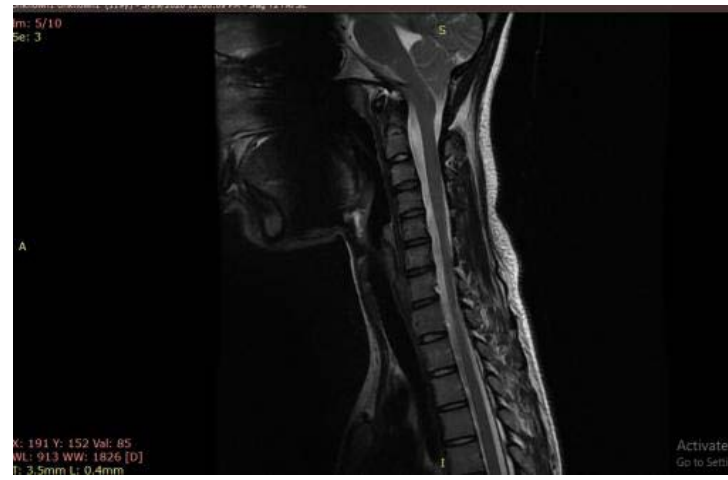

Figure 3: Sagittal T2WS image shows thinning (arrow) of lower cervical cord (C6-D1) with loss of normal curvature of the spine.

For evaluating loss of attachment between posterior dura and lamina, the lamina was defined as between junction of lamina medially and laterally by a tangential line along the medial aspect of the pedicle. This was divided equally into three parts. More than $33.3 \%$ loss of attachment between dura and lamina was significant [10].

\section{Observations}

\section{Clinical features}

The illness was insidious in onset with a duration of illness between fifteen to twenty months. Most common clinical findings were atrophy, weakness of grip, and tremors. Some patients had wasting of muscles of the forearm. All patients had lower motor neuron type of weakness.

\section{Imaging findings}

Lower cervical cord atrophy (Figure 3), anterior dural shift with flexion, and postcontrast enhancement of epidural component were found in thirteen out of thirteen patients (Figure 2 and 5) (100\%), followed by the hyperintense signal in T2WS in the cord which was found in 11 patients (84.6\%) (Figure 1). Loss of attachment of posterior dura (Figure 1,2 and 4) and subjacent lamina and abnormal curvature of the spine was found in 9 out of thirteen patients (69.3\%), prominent epidural flow void was found in eight out of thirteen patients (61.5\%), the thoracic extension of enhancing epidural component was found in five patients (38.4\%). The thickness of the enhancing epidural component was between 3-8.6 $\mathrm{mm}$ (Table 1).

\section{Discussion}

In a normal spine dura is a thin loose sheath with its attachment at the foramen magnum, dorsal surface of $\mathrm{C} 2$ and $\mathrm{C} 3$, and coccyx. Hirayama disease is a rare entity related to flexion of the cervical spine. The pathogenesis of this entity is debated. It is postulated to be caused by the anterior displacement of the dura during flexion with secondary compression of the lower cervical cord (C4-D1) and injury to anterior horn cells [11]. Normally during flexion increase in the length of the spine is compensated by transverse dural folds

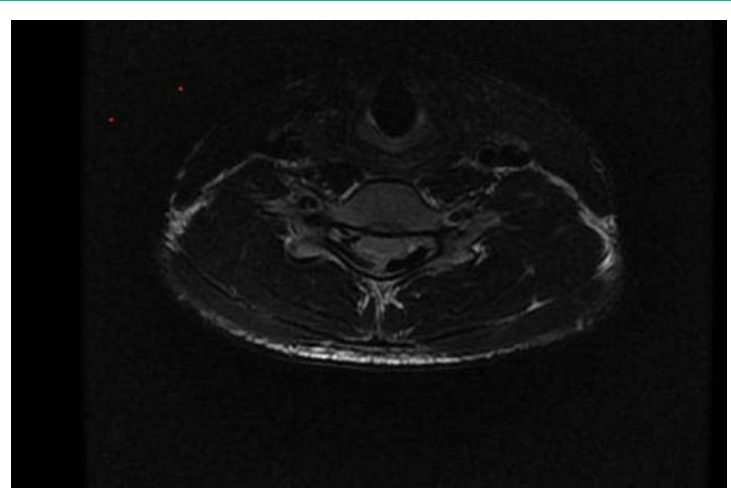

Figure 4: Axial T2WS image shows flow void in posterior epidural space (block arrow).

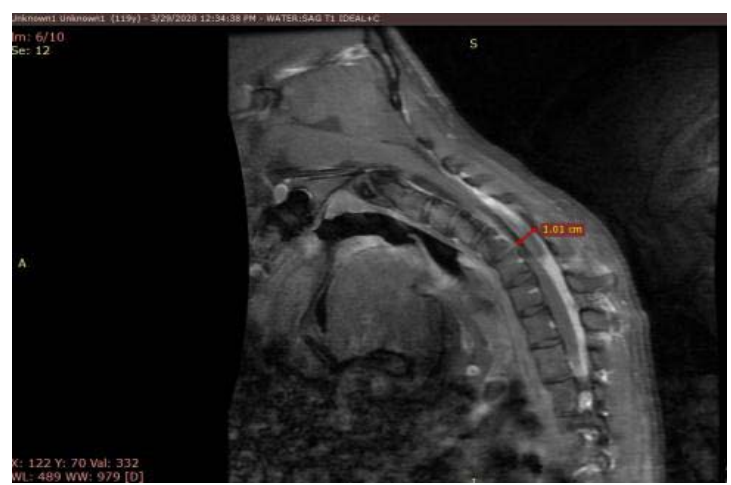

Figure 5: Flexion T1WFS shows enhancing epidural space (arrow). 


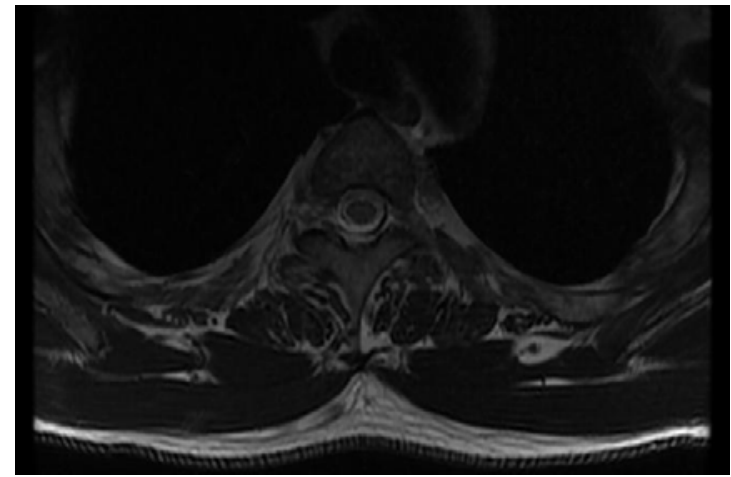

Figure 6: Axial T2WS image shows the detachment of posterior dura from subjacent lamina (arrow).

Table 1:

\begin{tabular}{|l|c|}
\hline \multicolumn{1}{|c|}{ Magnetic Resonance Imaging Features } & Number of Patients \\
\hline Localized lower cervical cord atrophy & 13 \\
\hline Anterior shift of posterior wall of cervical dura & 13 \\
\hline Enhancing epidural component & 13 \\
\hline Increased cord signal on T2WS & 11 \\
\hline Loss of attachment between posterior dura and lamina & 9 \\
\hline Abnormal cervical curvature & 9 \\
\hline Asymmetrical cord flattening & 6 \\
\hline Thoracic extension of enhancing epidural component & 5 \\
\hline Prominent epidural flow void & 8 \\
\hline
\end{tabular}

however in patients with Hirayama disease it is postulated that The disproportionate growth between the spinal cord and vertebral column causes short and tight dural sac and anterior displacement of posterior dura during flexion of neck $[7,12]$. In pathological studies, microcirculatory damage is the most common finding [11]. A study by Toma $\mathrm{S}$ and shiozawa proposed that disproportionate shortening of the dural sac in an accentuated juvenile growth spurt [13]. In a study done by Kira J, Ochi $\mathrm{H}$ in 2001 correlation was found between atopic disorders, level of IgE, and Hirayama disease [14].

The typical presentation is with progressive upper limb weakness, tremors, and atrophy in a young male with abnormal cervical spine curvature [15].

MRI findings suspicious for this condition is a focal increase in signal intensity in the lower part of cervical cord with asymmetrical thinning and anterior displacement of posterior dura, enhancing epidural component in the lower cervical and thoracic region and prominent posterior epidural flow voids suggestive dilated epidural venous plexus. . Anterior displacement of the dura with prominent epidural flow voids are considered as most specific finding [15]. For confirmation of the anterior shift of dura flexion-extension imaging with contrast can be done. There is a prominent venous flow void in the flexion image.

The anterior dural shift becomes less prominent with the chronic stage of the disease and with old age. In a postcontrast MRI scan, there is the crescent-shaped enhancement of posterior epidural space [15].
It is a self-limiting condition so treatment is only in form of cervical collar therapy however in some patient's surgical intervention which involves mainly cervical decompression and/or fusion with or without duraplasty [7]. As compared to a previous study done by HA sonwalker et al. in which the most common radiological finding in Hirayama disease was localized cord atrophy asymmetric cord flattening, enhancing epidural component, prominent posterior epidural flow voids, and anterior shifting of posterior wall of the dural canal [7]. In our study our study 13 out of 13 patients had localized cord atrophy, enhancing epidural component anterior shifting of dura however prominent epidural flow voids and asymmetric cord flattening was seen in 8 and 6 out of 13 patients respectively. Hence our findings were consistent with HA Sonwalker et al.

\section{Conclusion}

Dynamic postcontrast MRI plays a vital role in diagnosing Hirayama disease in clinically suspected cases.

\section{References}

1. Lehman VT, Leutmer PH, Sorenson EJ, et al. Cervical spine MR imaging findings of patients with Hirayama Disease in North America: A multisite study. AJNR. 2013; 32: 451-456.

2. Hirayama K, Tsubaki T, Toyokura Y. Juvenile muscular atrophy of unilateral upper extremity -a new clinical entity. Pshychiatr Neurol Jpn. 1959; 61: 21902198.

3. Narayan Gowda BS, Mohan Kumar J, Basim PK. Hirayama's Disease- A rare case report with review of literature. J Orthop Case Reports. 2013; 3: 11-14.

4. Tashiro K, Kikuchi S, Itoyama Y. Nationwide Survey of Hirayama Disease in Japan. Amyotroph Lateral Scler. 2006; 7: 38-45.

5. Hirayama K. Juvenile muscular atrophy of unilateral upper extremity -Halfcentury progress and establishment since its discovery. Brain Nerve. 2008; 60: 17-29.

6. Monali R, Rima K, et al. MRI findings in Hirayama disease.Indian J Radiol Imaging. 2010; 20: 245-249.

7. Sonwalkar HA, Shah RS, Khan Firosh K, et al. Imaging Features of Hirayama Disease. Neurol India. 2008; 56: 22-26.

8. Guigui $\mathrm{P}$, Benoist M.,Deburge A. Spinal deformity and instability after multilevel cervical laminectomy for spondylotic myelopathy. Spine (Phila $\mathrm{Pa}$ 1976). 1998; 23: 440-447.

9. Batzdorff $U$, Batzdorff $A$. Analysis of cervical spine curvature in patients with cervical spondylosis. Neurosurgery. 1988; 22: 827-836.

10. Chen CJ, Hsu HL, et al. Hirayama Flexion Myelopathy: Neutral Position MR imaging findings- Importance of attachment. Radiology. 2004; 231: 39-44.

11. Kikuchi S, Tashiro K. A mechanism of juvenile muscular atrophy localized in hand and forearm...flexion myelopathy with tight dural sac in flexion. 1987.

12. Toma S, Shiozawa Z. Journal of neurology, neurosurgery and pshychiatry. 1995; 58.

13. Kira J, Ochi H. Juvenile Muscular Atrophy of Distal Upper limb associated with Atopy. J neurol neurosurg pshychiatry. 2001; 70: 798-801.

14. De Jong. Handbook of Clinical Neurology. Elsevier: Amsterdam, the Netherlands; 1991; 15: 107-120.

15. Hirayama disease: MRI spectrum of a rare disease...sagar maheshwari, shweta shendey....eurorad. 2019 